\title{
Um resgate histórico do LABIOMEC na história do CEFD 50 anos: parte 1
}

\author{
A historical rescue of the LABIOMEC in the history of CEFD 50 years: part 1 \\ Un rescate histórico del LABIOMEC en la historia de CEFD 50 años: part 1
}

\begin{abstract}
Carlos Bolli Mota ${ }^{\mathrm{I}}$, Juliana Corrêa Soares ${ }^{\mathrm{II}}$, Carla Emilia Rossato ${ }^{\mathrm{III}}$, Franciele Marques Pivetta ${ }^{\mathrm{IV}}$, Marta Cristina Rodrigues da Silva ${ }^{\mathrm{V}}$, Mateus Corrêa Silveira ${ }^{\mathrm{VI}}$, Gabriela dos Santos de Souza ${ }^{\mathrm{VII}}$, Estele Caroline Welter Meereis-Lemos ${ }^{\mathrm{VIII}}$, Igor Martins Barbosa ${ }^{\mathrm{IX}}$, Rose Löbell ${ }^{\mathrm{X}}$, Rafaela Oliveira Machado ${ }^{\mathrm{XI}}$, Michele Forgiarini Saccol ${ }^{\mathrm{XII}}$
\end{abstract}

\section{Resumo}

Este trabalho tem como objetivo relatar um pouco da história e da evolução do Laboratório de Biomecânica (LABIOMEC) ao longo dos 50 anos do Centro de Educação Física e Desportos (CEFD) da Universidade Federal da Santa Maria. Para tanto foram realizadas buscas em documentos físicos e arquivos digitais no período de 1970 até os dias atuais em relação ao LABIOMEC em artigos, trabalhos em eventos, monografias de graduação, especialização, dissertações e teses. $\mathrm{O}$ artigo foi subdividido em duas partes e nesta primeira é realizado o resgate histórico dos anos iniciais e a evolução técnico-científica ocorrida. A importância desse relato é permitir que parte da história do LABIOMEC e das pessoas que realizaram suas atividades no laboratório como docentes ou discentes fique registrada de forma permanente.

Palavras-chave: Biomecânica; Educação Física e Treinamento; Movimento

\begin{abstract}
The purpose of this work is to report a little of the history and evolution that the Laboratory of Biomechanics (LABIOMEC) experienced over the 50 years of the Center for Physical Education and Sports (CEFD) of the Federal University of Santa Maria. For that purpose, searches were carried out on physical documents and digital files from 1970 to the present day in relation to LABIOMEC in articles, works in events, graduation monographs, specialization, dissertations and theses. The article was subdivided in two parts and in this first one, the historical recovery of the initial years is carried out. The importance of this report is to allow part of the history of LABIOMEC and the people who carried out their activities in the laboratory as teachers or students to be permanently registered.
\end{abstract}

Keywords: Biomechanics; Physical Education and Training; Movement

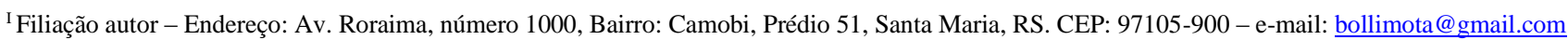

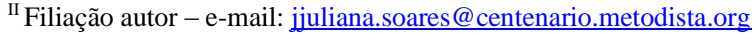

III Filiação autor - e-mail: carlinharossato@gmail.com

IV Filiação autor - e-mail: fran87.mp@ gmail.com

v Filiação autor - e-mail: martacrys1605@gmail.com

${ }^{\mathrm{V}}$ Filiação autor - e-mail: mm.biomec@ gmail.com

VII Filiação autor - e-mail: ggabrielassouza@gmail.com

VIII Filiação autor - e-mail: estelemeereis@gmail.com

IX Filiação autor - e-mail: igormartinsbarbosa2@gmail.com

x Filiação autor - e-mail: roselobell@gmail.com

XI Filiação autor-e-mail: rafa_omachado@hotmail.com

XII Filiação autor - e-mail: mfsaccol@gmail.com
} 


\section{Resumen}

El propósito de este trabajo es informar un poco de la historia y evolución que experimentó el Laboratorio de Biomecánica (LABIOMEC) durante los 50 años del Centro de Educación Física y Deportes (CEFD) de la Universidad Federal de Santa María. Para ello, se realizaron búsquedas en documentos físicos y archivos digitales desde 1970 hasta la actualidad en relación con LABIOMEC en artículos, trabajos en eventos, monografías de graduación, especialización, disertaciones y tesis. El artículo fue subdividido en dos partes y em esta primera se realiza la recuperación histórica de los años iniciales y la evolución técnico-científica que se produjo. La importancia de esto informe es para permitir que parte de la historia de LABIOMEC y las personas que llevaron a cabo sus actividades en el laboratorio como maestros o estudiantes se registren permanentemente.

Palabras clave: Biomecánica; Educación y Entrenamiento Físico; Movimiento

\section{Introdução}

O termo biomecânica é a combinação do prefixo bio, que significa vida, com o termo mecânica, ramo da física que diz respeito ao estudo das ações das forças no movimento. Na década de 1970, pesquisadores internacionais adotaram o termo biomecânica para descrever a área do conhecimento que envolve o estudo dos aspectos mecânicos dos organismos vivos. Dentro dos campos da cinesiologia e da ciência do exercício, o organismo vivo mais comumente interessante é o corpo humano e as forças estudadas dentro da biomecânica incluem as forças internas produzidas principalmente pelos músculos e as forças externas que atuam no corpo humano (HALL, 2016). Nas décadas seguintes, a biomecânica foi desenvolvida como uma área de estudo nos currículos de graduação e pós-graduação da América do Norte.

No Brasil, o desenvolvimento da biomecânica ocorreu a partir de um convênio entre o Brasil e a Alemanha em 1965, o que permitiu a vinda de professores para estabelecer as bases necessárias ao desenvolvimento dessa área nos cursos de Educação Física existentes no país (DIEM; 1983 apud ACQUESTA et al., 2007; AMADIO; SERRÃO, 2011). A origem do Laboratório de Biomecânica (LABIOMEC) está relacionada ao estabelecimento desse convênio.

\section{Histórico}

A história do LABIOMEC do Centro de Educação Física e Desportos da UFSM (CEFD) está intimamente relacionada à implantação e desenvolvimento da área de biomecânica no Brasil. Em 1965 foi realizado um convênio entre o Brasil e a República Federal da Alemanha para a introdução da Biomecânica nos cursos de Educação Física (DIEM; 1983 apud ACQUESTA et al., 2007; AMADIO; SERRÃO, 2011). Como parte desse convênio e buscando a formação de especialistas para ministrarem as aulas, o primeiro 
curso de especialização na área de biomecânica no Brasil foi realizado no ano de 1976 pelo Dr. Hartmut Riehle, na Escola de Educação Física da Universidade de São Paulo e no CEFD em Santa Maria (ACQUESTA et al., 2007; AMADIO; SERRÃO, 2011) (Figura 1). Entre os alunos dessa turma, estava o egresso da primeira turma do curso de Educação Física da UFSM e docente no CEFD, Professor Aluisio Otávio Vargas Avila.

Figura 1 - Fotografias do curso de Especialização em Biomecânica

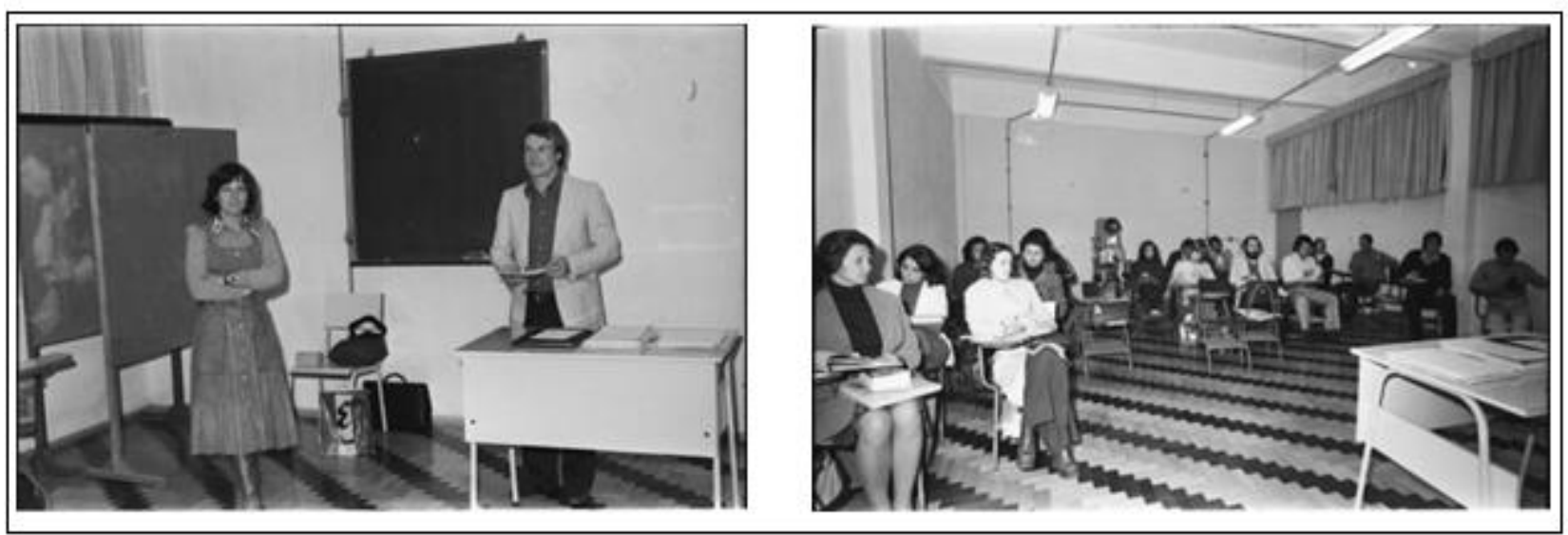

Fonte: Departamento de Arquivo Geral, Universidade Federal de Santa Maria, 2020.

Com a necessidade de ampliar a formação dos docentes no ensino superior e pesquisadores, em 1979 foi implantado na UFSM o Programa de Mestrado em Ciência do Movimento Humano, sendo o segundo programa criado no Brasil (SANTOS, 2012). Ainda como parte do acordo cultural, no período de 2 a 12 de setembro de 1980, o Prof. Dr. Wolfgang Baumann, então chefe do Institut für Biomechanik da Deutsche Sporthochschule Köln da Alemanha, juntamente com o Prof. Sepp Schönmetzler, ministrou um curso de Biomecânica no Programa de Pós-Graduação em Educação Física do CEFD. Além do curso, nessa visita também foi possível uma orientação especializada para construção de laboratórios que pudessem desenvolver estudos na área, elencando setores de instalação e distribuição de pessoal (BAUMANN; SCHÖNMETZLER; LENK, 1980).

Já em 4 de novembro de 1982, houve a criação do Laboratório de Pesquisa e Ensino do Movimento Humano (LAPEM) com os seguintes núcleos: Núcleo de Informática, Núcleo de Biomecânica, Núcleo de Fisiologia do Exercício, Núcleo de Aprendizagem Motora e Núcleo de Pedagogia e Pesquisa Curricular (Figura 2). Entretanto, o desenvolvimento efetivo do LAPEM ocorreu a partir de 1984, com o retorno ao CEFD dos professores que realizaram seus cursos de Mestrado e Doutorado no exterior. Entre eles, o Professor Aluisio que desenvolveu na Vanderbilt University, no período de 1979 a 1983, suas pesquisas em biomecânica com os títulos Biomechanical analysis of vaulting (mestrado) e A comparative study of three methods of determining the center of gravity of the human body (doutorado) (Figura 3). 
Figura 2 - Logotipo do Laboratório de Pesquisa e Ensino do Movimento Humano (LAPEM) (A), primeiro logotipo do Laboratório de Biomecânica (LABIOMEC) (B) e o logotipo atual do LABIOMEC (C).

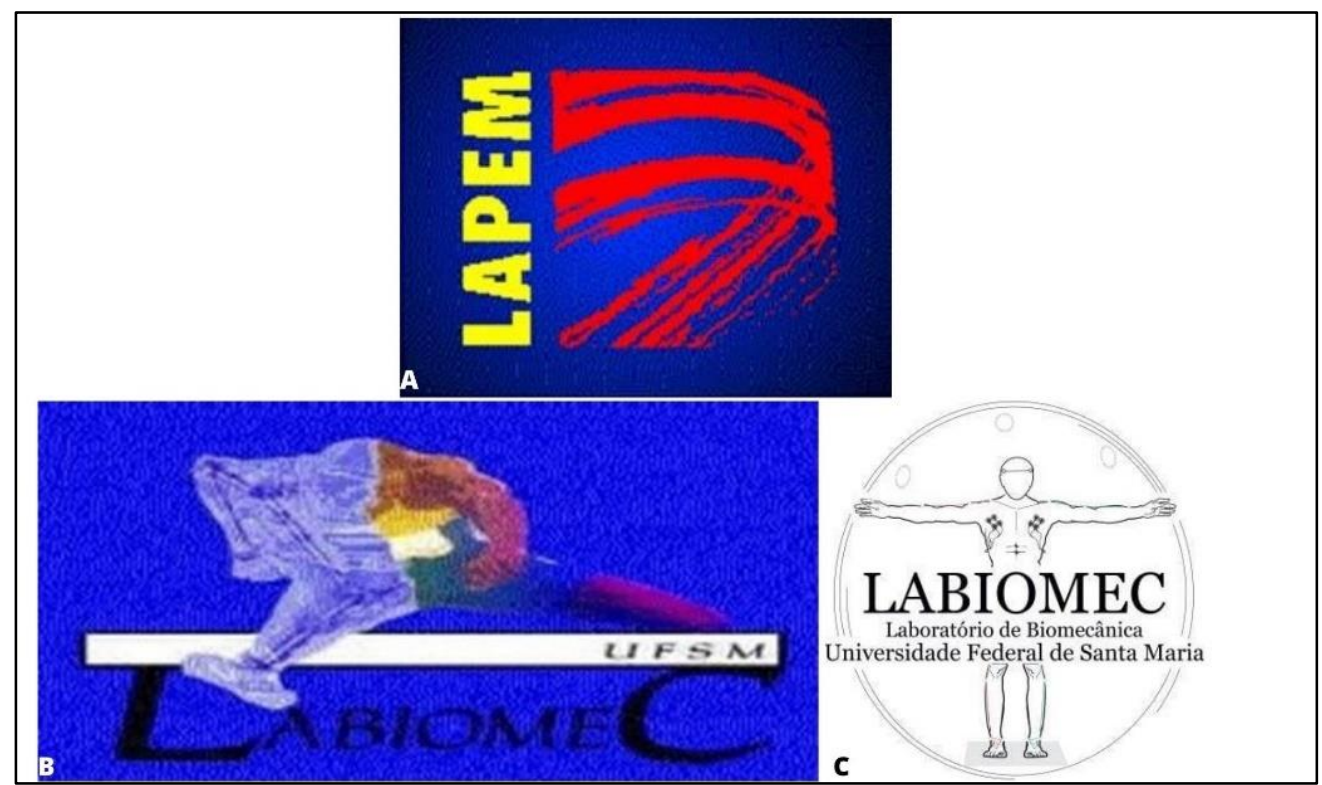

Fonte: Arquivo do LABIOMEC

Figura 3 - Análise biomecânica do salto sobre o cavalo (front-handspring-vault): filmagem com câmera Bolex Paillard 16 $\mathrm{mm}$, frequência de aquisição de $64 \mathrm{~Hz}$ (A); desenho esquemático das fases do salto (B), e trajetória do centro de gravidade do salto analisado em folha milimetrada $(\mathrm{C})$.
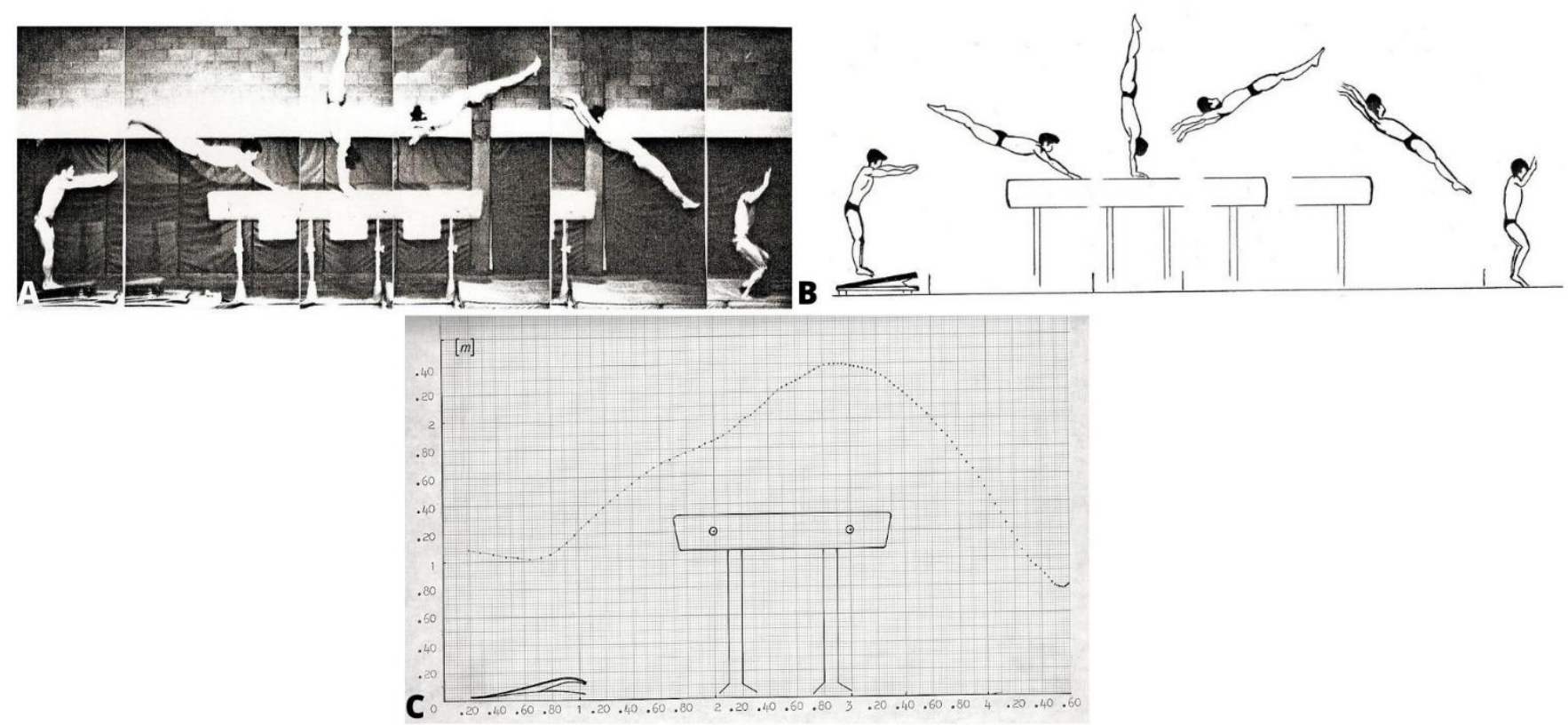

Fonte: AVILA, 1983. 
Como docente no período de 1973 a 1998, o Professor Aluisio foi o primeiro responsável pelas atividades do laboratório. Para atender às necessidades da pesquisa, o laboratório procurou formar um grupo interdisciplinar, buscando profissionais de diferentes áreas do conhecimento. O engenheiro eletricista Noé Gomes Borges Júnior, o engenheiro mecânico Carlos Bolli Mota e o médico cardiologista Paulo Tadeu Cavalheiro integraram o grupo de pesquisadores. Entre as pesquisas e projetos realizados nesse período destacam-se a análise de movimentos esportivos, o desenvolvimento de protótipos e softwares, e a biomecânica do pé e do calçado.

A análise de movimentos esportivos ocorreu em dissertações de mestrado com o tema de ginga da capoeira (RODRIGUES, 1988), saltos pliométricos (NASSER, 1990) e em distância (ROCHA JUNIOR, 1993). Esse último refere-se à dissertação de mestrado do Prof. Ivon Chagas da Rocha Junior, que também colaborou com os trabalhos e atividades do LABIOMEC ao longo de sua docência no CEFD (1993-2017). Esses primeiros trabalhos com cinemetria no laboratório foram realizados com a técnica de cinematografia bidimensional plana, utilizando câmeras analógicas (tipo LOCAM-51) e posterior revelação dos filmes para digitalização manual dos dados (mesa digitalizadora Digicon MDD 1912). O desenvolvimento dos primeiros softwares e programas específicos do LABIOMEC para análise de dados da cinemetria também tiveram início nessa época (Figura 4).

Figura 4 - Pontos referentes aos marcadores anatômicos (A), modelo corporal reconstruído a partir dos pontos dos eixos articulares (B), centros de massa dos segmentos (C) e trajetória do centro de gravidade total do corpo (D) desde o instante da impulsão da terceira última passada até o instante da impulsão final no salto em distância.

A

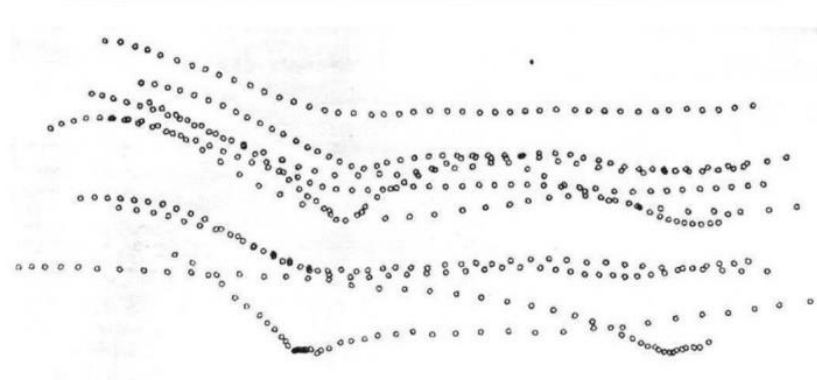

B
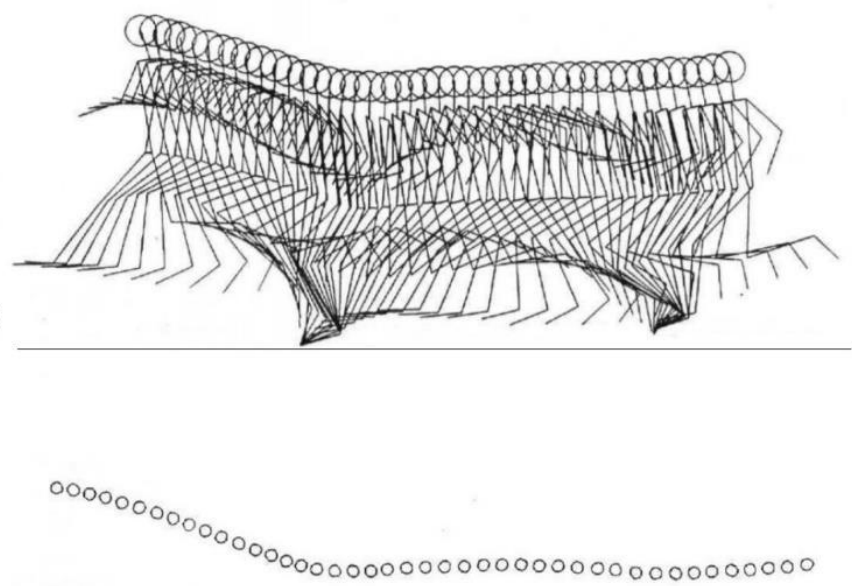

C

D 
Além do desenvolvimento de softwares para análise de dados dos trabalhos do grupo, a dificuldade na aquisição e mesmo a inexistência de alguns dispositivos de análise despertaram o desenvolvimento de protótipos e métodos biomecânicos. Entre os projetos desenvolvidos estão a construção de uma placa interface digital para eletrocardiograma com oito canais simultâneos (BORGES JUNIOR, 1991), plataforma de força digital, deslizômetro linear digital, tapete para cronometragem de tempos do teste de potência de Margaria-Kalamen, um protótipo de simulador de posturas sentadas para análise ergométrica (MELLO, 1994; MORO, 1994) (Figura 5 e 6) e um sistema de avaliação do coeficiente de atrito entre calçados esportivos e pisos (MELO, 1995) (Figura 7). Esse último projeto tem relação com umas das linhas de pesquisa e de trabalho de maior repercussão do laboratório: o estudo antropométrico do pé brasileiro.

Figura 5 - Protótipo de estações de trabalho equipado com célula de carga para avaliar posições assumidas e níveis de conforto em digitadores na situação A (cadeira gravidade zero) e B (cadeira de escritório convencional).

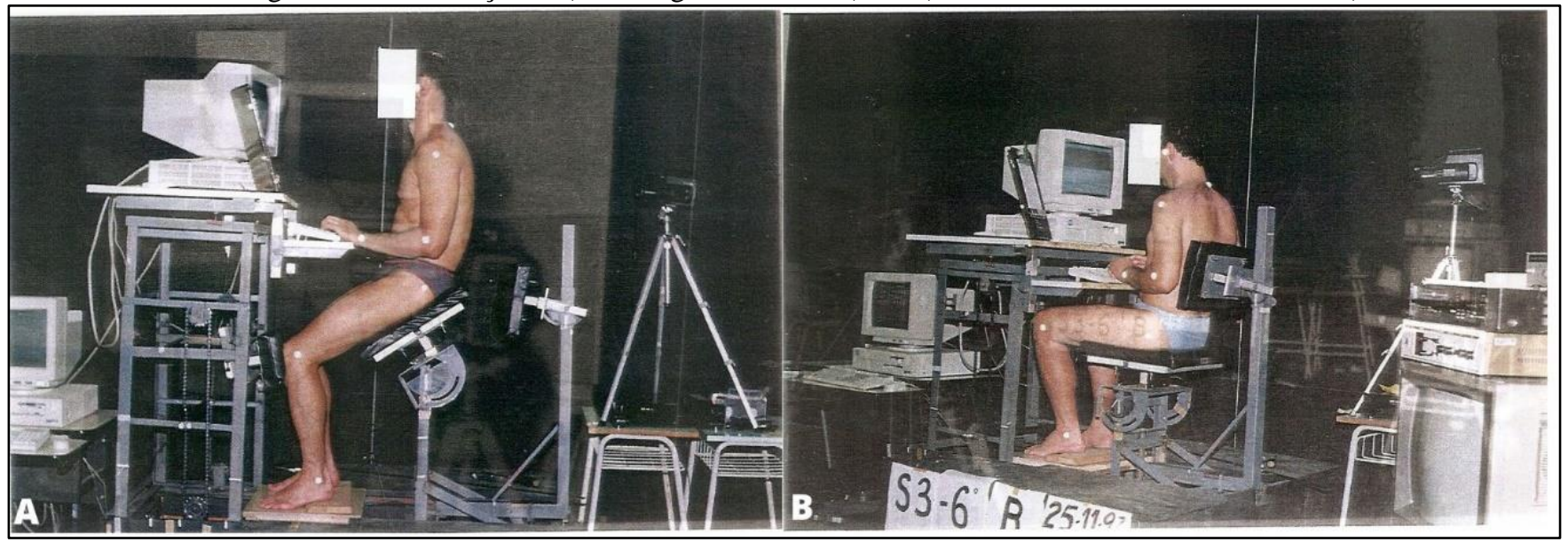

Fonte: MELLO, 1994.

Um dos principais focos de pesquisa do professor Aluisio foi a biomecânica do pé e do calçado. Uma grande pesquisa em nível nacional buscava determinar os padrões dimensionais dos pés da população brasileira e a criação de um banco de dados nacional. Em um esforço conjunto, o Centro Tecnológico do Couro, Calçados e Afins e o LAPEM propuseram um estudo antropométrico-biomecânico dos pés brasileiros e a definição de parâmetros técnicos para a fabricação de calçados que atendessem as condições de saúde e conforto. A proposta contribuiu para a indústria do Rio Grande do Sul, permitindo a criação de tecnologias de ponta e tornando-a competitiva no mercado calçadista. Os primeiros trabalhos relativos a essa pesquisa avaliaram as características dinâmicas do caminhar humano em função do calçado (MACHADO, 1994), os parâmetros antropométricos e biomecânicos do pé para fabricação de calçados (MANFIO, 1995) (Figura 8) e a variação do arco plantar longitudinal com diferentes alturas de apoio do calcâneo (NASSER; AVILA, 2000). 
Figura 6 - Protótipo equipado com célula de carga para comparar distribuição do peso corporal em sujeito executando tarefa de leitura em três situações experimentais de mobiliário cadeira-mesa: assento inclinado à frente em $30^{\circ}$, apoio para joelhos e tampo da mesa inclinado em $15^{\circ}(\mathrm{A})$; mesma posição anterior, mas tampo da mesa na horizontal (B); assento e tampo na horizontal, suporte para apoio das costas (C).

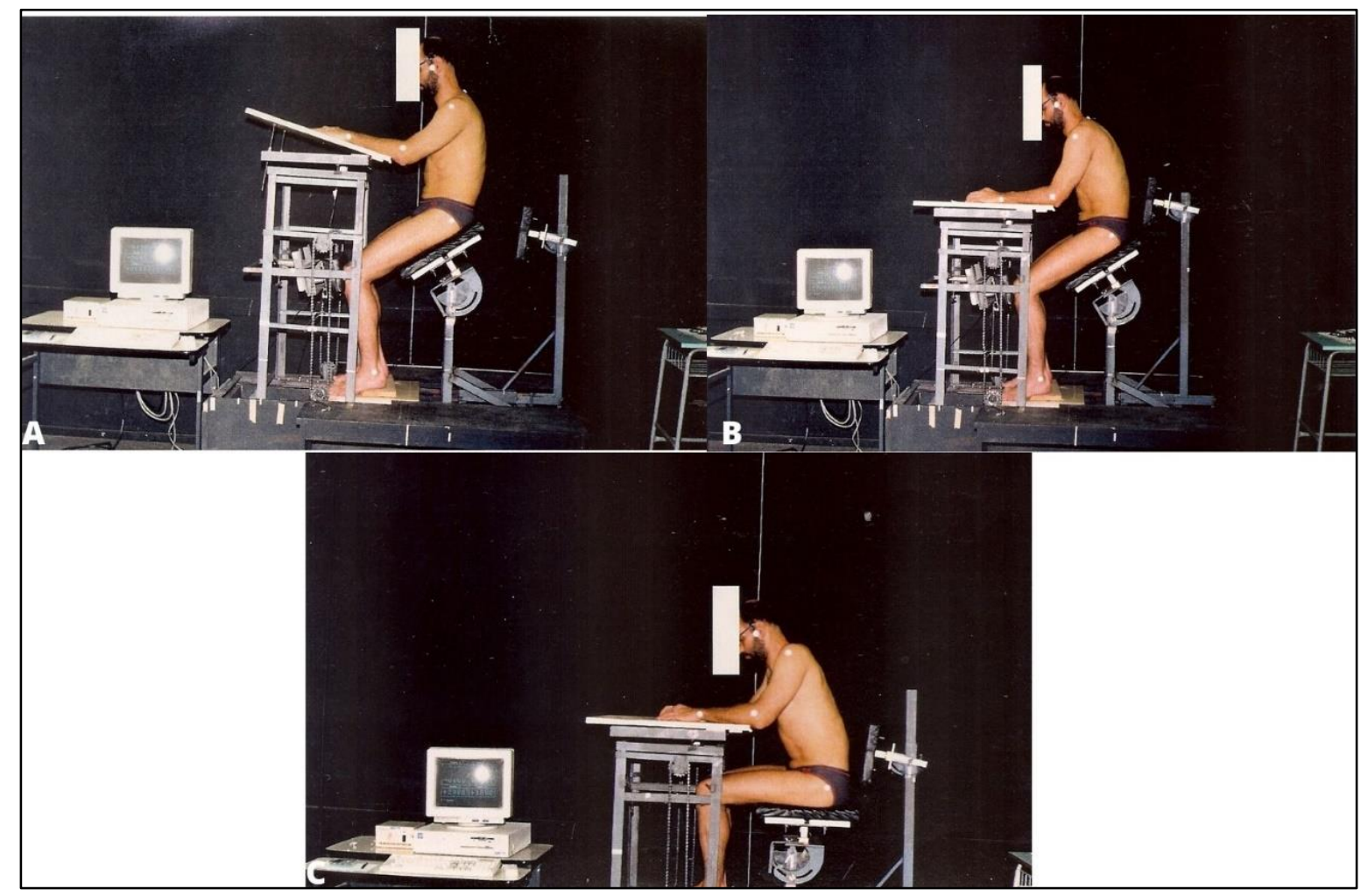

Fonte: MORO, 1994.

Figura 7 - Desenho do sistema de elevação e detecção do início do deslize automatizados e protótipo da versão automatizada para avaliação do coeficiente de atrito entre calçados esportivos e pisos.

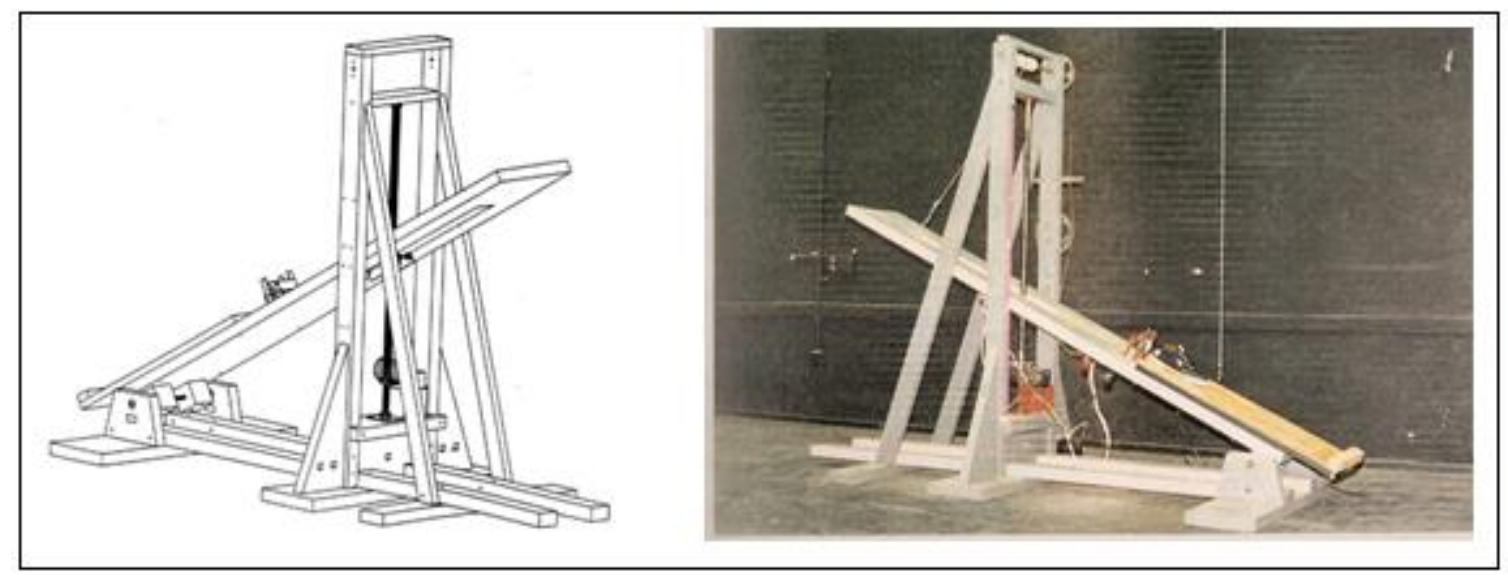

Fonte: MELO, 1995. 
Figura 8 - Variáveis antropométricas avaliadas no estudo do pé brasileiro: comprimentos e ângulos (A), variáveis de larguras (B), variáveis de perímetros (C) e variáveis de altura (D).

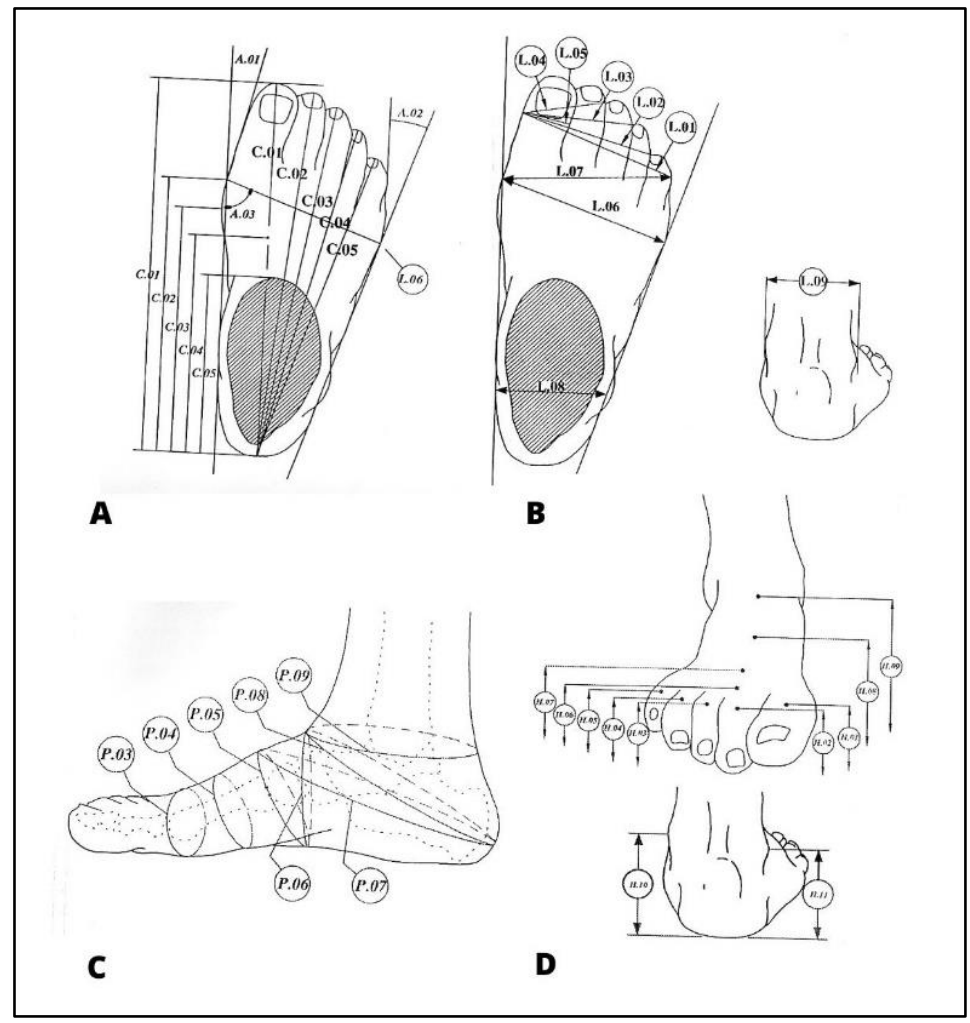

Fonte: MACHADO, 1994.

Como esse projeto temático envolvia medir um número significativo de pés e a medida direta se mostrava inviável, a solução mais adequada seria fotografar os pés, um processo rápido, e obter as medidas posteriormente a partir das imagens. A tese de doutorado "Concepção e implementação de um sistema de medição do pé humano baseado em processamento digital de imagens"“ consistiu no desenvolvimento e testagem de um software completo para obter as medidas de interesse a partir de imagens fotográficas dos pés (Figura 9) (MOTA, 1999). A tese foi desenvolvida pelo técnico administrativo e engenheiro mecânico Carlos Bolli Mota, que ao longo de seu doutorado foi aprovado como Professor Assistente do CEFD em 1994.

A partir da década de 90, a aquisição de equipamentos como o sistema de palmilhas de análise de pressão plantar (F-scan), sistema de cinemetria (Peak Motus), plataforma de força (AMTI) e posteriormente eletromiógrafo (EMG Lynx) permitiram o desenvolvimento de projetos e pesquisas com maior precisão de análise. 
Figura 9 - Medidas do processamento digital de imagens do pé humano com treze comprimentos, três larguras e dois ângulos dos pés.

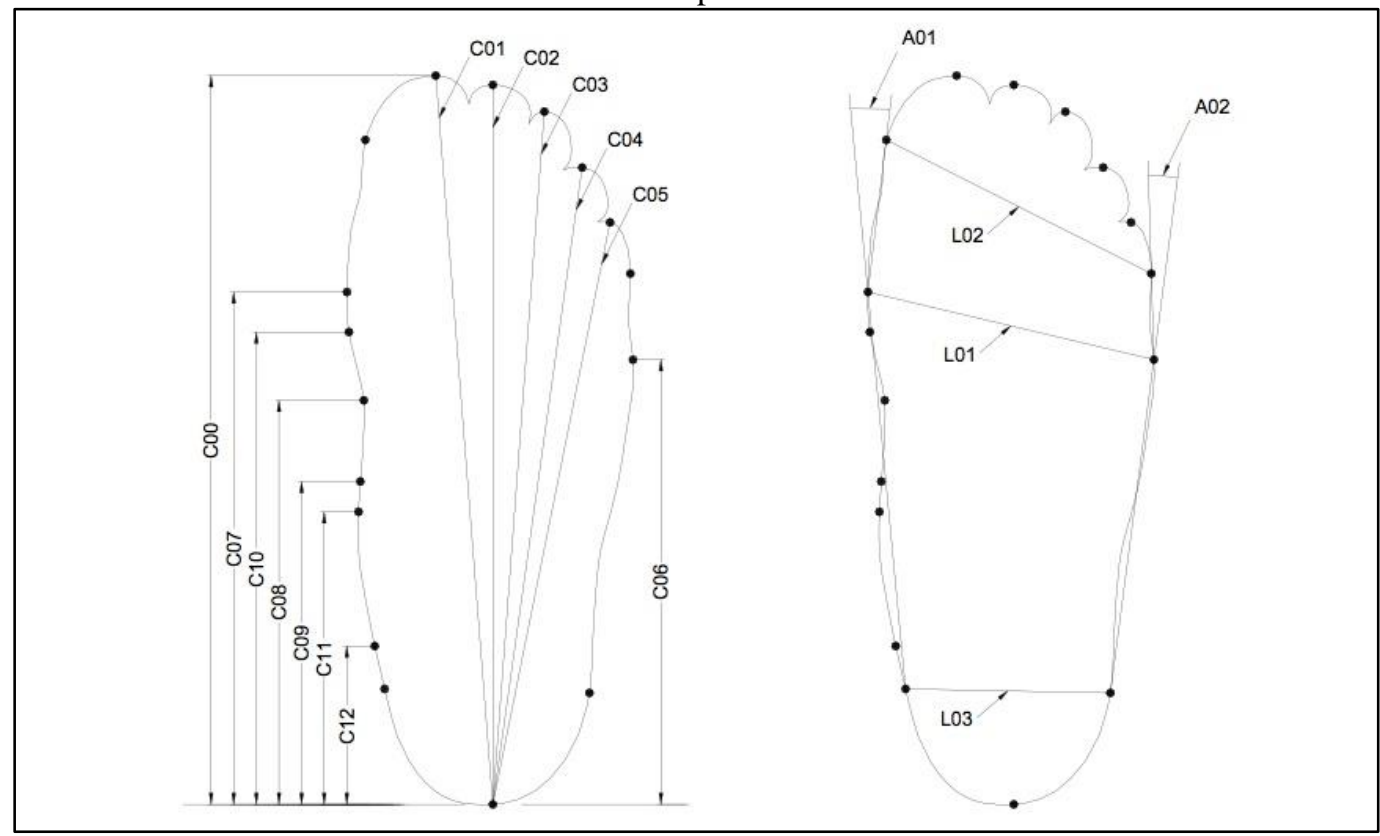

Fonte: MOTA, 1999.

\section{Conclusão}

Nesta primeira parte do resgate histórico do LABIOMEC foi relatado como ocorreu o desenvolvimento da área de biomecânica no Brasil, bem como os anos iniciais do laboratório e a evolução técnico-científica ocorrida. Em uma segunda parte do artigo, serão apresentados os principais trabalhos desenvolvidos no LABIOMEC a partir da década de 90, nas linhas de pesquisa de cinemática e cinética, controle postural e eletromiografia.

\section{Referências}

ACQUESTA, F. M et al. O estudo da biomecânica do movimento humano no brasil através da análise da distribuição das publicações da Revista Brasileira de Biomecânica no período 2000-2006. Revista Brasileira de Biomecânica, v. 8, n. 15, p. 67-73, 2007. Disponível em: http://citrus.uspnet.usp.br/biomecan/ojs/index.php/rbb/article/viewFile/67/40. Acesso em: 30 maio 2020.

AMADIO, A. C.; SERRÃO, J. C. A Biomecânica em Educação Física e esporte. Revista Brasileira de Educação Física e Esporte, v. 25, p.15-24, 2011. 
AVILA, A. O. V. A comparative study of three methods of determining the center of gravity of the human body. 1983. 120 f. Tese (Doutorado em Saúde e Educação Física), Vanderbilt University, Estados Unidos, 1983.

BAUMANN, W.; SCHÖNMETZLER, S.; LENK, M. Curso de Biomecânica. Santa Maria-RS: Universidade Federal de Santa Maria. 1980.

BORGES JUNIOR, N. G. Projeto de um eletrocardiograma segundo uma nova concepção. 1991. $84 \mathrm{f}$. Dissertação (Mestrado em Ciência do Movimento Humano), Universidade Federal de Santa Maria, Santa Maria, 1991.

HALL, S. J. Biomecânica Básica. Rio de Janeiro: Guanabara Koogan. 2016.

MACHADO, D. B. Estudo das características dinâmicas do caminhar humano, em função do calçado. 1994. 100 f. Dissertação (Mestrado em Ciência do Movimento Humano), Universidade Federal de Santa Maria, Santa Maria, 1994.

MANFIO, E. F. Estudo de parâmetros antropométricos e biomecânicos do pé humano para a fabricação de calçados segundo critérios de conforto, saúde e segurança. 1995. 50 f. Dissertação (Mestrado em Ciência do Movimento Humano), Universidade Federal de Santa Maria, Santa Maria, 1995. Disponível em: http://search.ebscohost.com/login.aspx?direct=true \&db=cat00956a\&AN=ufsm.661707\&site=eds-live. Acesso em: 30 maio 2020.

MELLO, O. D. S. Análise da postura do digitador em situações experimentais utilizando um protótipo denominado "simulador de posturas sentadas". 1994. 50 f. Dissertação (Mestrado em Ciência do Movimento Humano), Universidade Federal de Santa Maria, Santa Maria, 1994. Disponível em: http://search.ebscohost.com/login.aspx?direct=true \&db=cat00956a\&AN=ufsm.685395\&site=eds-live.

Acesso em: 30 maio 2020.

MELO, S. I. L. Um sistema para determinação do coeficiente de atrito (u) entre calçados esportivos e pisos usando o plano inclinado. 1995. 100 f. Tese (Doutorado em Educação Física), Universidade Federal de Santa Maria, Santa Maria, 1995. Disponível em: http://search.ebscohost.com/login.aspx?direct=true \&db=cat00956a\&AN=ufsm.661699\&site=eds-live.

Acesso em: 30 maio 2020.

MORO, A. R. P. Distribuição do peso corporal do sujeito na posição sentado: um estudo de três situações experimentais simuladas por um protótipo. 1994. 100 f. Dissertação (Mestrado em Ciência do Movimento Humano), Universidade Federal de Santa Maria, Santa Maria, 1994.

MOTA, C. B. Concepção e implementação de um sistema de medições do pé humano baseado no processamento digital de imagens. 1999. 100 f. Tese (Doutorado em Ciência do Movimento Humano), Universidade Federal de Santa Maria, Santa Maria, Santa Maria, 1999. 
NASSER, J. P. Análise de variáveis do salto pliométrico através dos métodos cinematográficos e dinamográficos. 1990. 50 f. Dissertação (Mestrado em Ciência do Movimento Humano), Universidade Federal de Santa Maria, Santa Maria, 1990.

NASSER, J. P.; AVILA, A. O. V. Estudo da variação do arco plantar longitudinal com apoio do calcâneo em diferentes alturas. Kinesis Santa Maria, v. 23, p. 115-138, 2000.

ROCHA JUNIOR, I. C. Análise cinemática do salto em distância: fase final da corrida e impulsão. 1993. 55 f. Dissertação (Mestrado em Ciência do Movimento Humano), Universidade Federal de Santa Maria, Santa Maria, $1993 . \quad$ Disponível em: http://search.ebscohost.com/login.aspx?direct=true $\& d b=$ cat00956a\&AN=ufsm.699606\&site=eds-live.

Acesso em: 30 maio. 2020.

RODRIGUES, S. C. P. Análise biomecânica da ginga na capoeira. 1988. 55 f. Dissertação (Mestrado em Ciência do Movimento Humano), Universidade Federal de Santa Maria, Santa Maria, 1988. Disponível em: http://search.ebscohost.com/login.aspx?direct=true \&db=cat00956a\&AN=ufsm.743581\&site=eds-live.

Acesso em: 30 maio. 2020.

SANTOS, J. D. S. A produção do conhecimento em educação física: análise epistemológica das dissertações e teses dos Programas de Pós-Graduação nas universidades públicas da região sul do Brasil (2000-2010). 2012. 120 f. Tese (Doutorado em Educação), Universidade Estadual de Campinas, CampinasSP, $2012 . \quad$ Disponível em: http://repositorio.unicamp.br/bitstream/REPOSIP/251033/1/Santos_JoaoDerlideSouza_D.pdf. Acesso em: 30 maio 2020.

UFSM. Universidade Federal de Santa Maria. Departamento de Arquivo Geral. Disponível em: http://fonte.ufsm.br/index.php/curso-de-pos-graduacao-em-biomecanica-1. Acesso em: 30 maio 2020.

\section{Como citar este artigo}

MOTA, C B; SOARES, J. C; ROSSATO, C. E; PIVETTA, F. M; SILVA, M. C. R; SILVEIRA, M. C; SOUZA, G. S; MEEREIS-LEMOS, E. C. W; BARBOSA, I. M.; LÖBELL, R; MACHADO, R. O; SACCOL, M.F. Um resgate histórico do LABIOMEC na história do CEFD 50 anos: parte 1. Revista Kinesis, Santa Maria, Dossiê CEFD 50 anos, p.01-11, 2020.

*O presente trabalho não contou com apoio financeiro de nenhuma natureza para sua realização. 